\title{
A Retrospective Study of Biliary Drainage Strategies for Patients with Malignant Hilar Biliary Strictures
}

\author{
Xin-Yue Liang ${ }^{1,2}$ \\ Wen Li iD ${ }^{1,2}$ \\ Fang Liu ${ }^{1,2}$ \\ Xin-Dan Kang (iD ${ }^{1,2}$ \\ 'Medical School of Chinese People's \\ Liberation Army (PLA), Beijing, People's \\ Republic of China; ${ }^{2}$ Department of \\ Gastroenterology and Hepatology, The \\ First Medical Center, Chinese PLA \\ General Hospital, Beijing, People's \\ Republic of China
}

\begin{abstract}
Purpose: The main aim of this study was to compare the efficacy and safety of different biliary drainage strategies, including percutaneous transhepatic biliary drainage (PTBD) versus endoscopic biliary stenting (EBS) and unilateral versus bilateral stenting, in patients with unresectable malignant hilar biliary strictures (MHBSs).

Patients and Methods: This was a retrospective review of patients with inoperable MHBSs who underwent biliary drainage by either EBS or PTBD. Efficacy and safety were compared between the two pathways and between unilateral and bilateral stenting in the EBS group. The survival duration was analyzed with K-M curves and Log rank tests.

Results: From January 2015 to December 2019, a total of 206 (126: EBS and 80: PTBD) patients with MHBSs were enrolled in our study and underwent 270 procedures (173: EBS and 97: PTBD). Bilateral stenting was superior to unilateral stenting in terms of clinical success $(69.6 \%$ vs $50.6 \%, p=0.039)$, especially for patients with Bismuth type IV $(70.0 \%$ vs $30.3 \%, \mathrm{p}=0.002$ ). A higher decrease in bilirubin was seen with PTBD in patients with Bismuth types III-IV (66.9 vs $36.7, \mathrm{p}=0.006$ ). A survival advantage was seen in successful drainage (227 days vs 82 days, $\mathrm{p}<0.001$ ), lower tumor-node-metastasis (TNM) scores (I-II) (195 days vs 139 days, $\mathrm{p}=0.012$ ), and cholangiocarcinoma ( 184 days vs 84 days, $\mathrm{p}=0.001$ ). Conclusion: For patients with advanced MHBSs, bilateral stenting may achieve a better drainage effect than unilateral stenting, and PTBD may have a better performance in relieving cholestasis than EBS. Successful drainage and cholangiocarcinoma may provide greater long-term survival benefits.
\end{abstract}

Keywords: inoperable hilar malignancy, endoscopic biliary stenting, percutaneous transhepatic biliary drainage, survival

\section{Introduction}

Malignant hilar biliary strictures (MHBSs) are formed mainly due to cholangiocarcinoma, gallbladder carcinoma, hepatocellular carcinoma and metastases or compression of metastatic lymph nodes. Curative resection is the only option associated with a chance of long-term survival. However, only $20-30 \%$ of patients are eligible for R0 resection once diagnosed. ${ }^{1}$ Unresectable malignant hilar strictures are associated with very poor prognosis, with a median survival of 5-9 months. ${ }^{2,3}$ For these patients, palliative biliary drainage should be offered to reduce bilirubin and preserve liver function. Endoscopic and percutaneous biliary drainage have been established as effective and relatively noninvasive decompression methods. For MHBSs with higher Bismuth types (III-IV), the superiority between percutaneous transhepatic biliary drainage (PTBD) and endoscopic biliary stenting (EBS) remains unresolved. Compared with PTBD, EBS can provide a better quality of life
Correspondence: Wen Li

Medical School of Chinese People's Liberation Army (PLA), Yard 28, Fuxing Street, Haidian District, Beijing, People's Republic of China

Tel +86 I39|I589|19

Fax +86 1055499307

Email liwen2000@yahoo.com 
for patients without requiring them to carry drainage catheters and bags. Moreover, EBS may be associated with a longer life expectancy. ${ }^{4}$ However, PTBD outperforms EBS with respect to relieving jaundice due to its more precise lobar selection with guidance of ultrasound and CT scans. In addition, the use of unilateral versus bilateral stenting is another heated discussion. Unilateral stenting is considered to be inadequate to achieve successful drainage, while bilateral stenting is technically challenging. To date, studies on palliative drainage for unresectable MHBS patients are sparse. The aim of this study was to compare the efficacy and safety of PTBD versus EBS and unilateral versus bilateral drainage in patients with inoperable MHBSs. In addition, survival assessments were performed for these patients.

\section{Materials and Methods}

\section{Patients}

Patients diagnosed with MHBSs between January 2015 and December 2019 were retrospectively screened in our center.

The inclusion criteria included (a) patients who underwent EBS or PTBD and (b) patients with inoperable MHBSs.

The exclusion criteria included patients with missing procedure information.

The diagnosis of stenosis in the hilum was established by abdominal computed tomography (CT), magnetic resonance imaging (MRI) and magnetic resonance cholangiopancreatography (MRCP). The diagnosis of malignancy was established by percutaneous transhepatic forceps biopsy, previous exploratory surgeries, or clinical findings (patient history, imaging findings, and neoplastic markers).

The collected data included demographic data, clinical data, imaging data, pathological data, types of treatment, postprocedure complications, adjuvant treatment, and follow-up data.

Bismuth classification was assigned by expert endoscopists based on a review of available radiologic and endoscopic imaging.

This study was approved by the Ethics Committee of the Medical School of Chinese People's Liberation Army (PLA). Informed consent was granted by all the participants included. The study was performed in accordance with the human and ethical principles of research set forth in the Helsinki guidelines.

\section{Biliary Drainage}

Endoscopic biliary drainage procedures were performed by experienced gastroenterologists in our center, and PTBD procedures were performed by experienced interventional radiologists.

PTBD referred only to external catheter drainage. Bilateral stent insertion was performed using the side-byside (SBS) technique.

ERCP procedures were performed with the patient in the supine position under sedation. A prophylactic treatment with broad-spectrum antibiotics was initiated before the procedure and continued for 3-5 days after. All endoscopic examinations were performed using a therapeutic duodenoscope (TJF-260, TJF-240, Olympus, Tokyo, Japan). The strategy used for biliary decompression was selected at the discretion of the endoscopists, based on the location and complexity of the strictures after discussing with patients before the procedure or their family members during the procedure. Self-expanding metal stents (COOK, USA) and plastic stents (COOK, USA) were inserted in our study.

\section{Follow-Up}

Patients were followed until death or the end of the study period (December 2020). The follow-up data were obtained from medical records or from telephone interviews with the patients or their families. The routine follow-up protocol included patient survival and adjuvant therapies after discharge.

\section{Definition}

Technical success (TS) was defined as placement of a biliary stent or drainage catheter in the setting of a stricture or the removal of biliary sludge without the need for additional stent placement (ie, no stricture).

Clinical success (CS) in a technically successful procedure was defined as a decrease in total bilirubin to less than $75 \%$ of the pretreatment value within a month.

An early complication was defined as any procedurerelated complication within 4 weeks, including cholangitis, hemorrhage, and postprocedure pancreatitis.

Cholangitis was defined as a body temperature of $\geq 38^{\circ}$ C, leukocytosis (white blood cell count $\geq 10 \times 100 / \mathrm{L}$ ), and worsening of liver function tests after the procedure.

Postprocedure pancreatitis was defined as follows: new or worsened abdominal pain with elevation of the serum amylase level to at least threefold greater than the upper limit of normal for 24 hours after the procedure. 
Hemorrhage was considered clinically significant only if there was clinical (not merely endoscopic) evidence of bleeding, such as melena or hematemesis, with an associated decrease in the hemoglobin concentration of at least $2 \mathrm{~g} / \mathrm{dL}$, or the need for a blood transfusion.

The tumor stage was determined by the eighth edition of the American Joint Committee on Cancer (AJCC) staging manual, and Memorial Sloan Kettering Cancer Center (MSKCC) staging was evaluated in patients with hilar cholangiocarcinoma. ${ }^{6}$

Survival was calculated from the day of the EBS or PTBD procedure.

\section{Statistical Analysis}

Statistical analysis was performed using SPSS 23.0. Quantitative data with a normal distribution were analyzed by the $t$-test and are presented as means; otherwise, they were analyzed by the Mann-Whitney $U$-test and are presented as medians. Qualitative data were analyzed using the chi-square test or Mann-Whitney $U$-test and are presented as ratios and frequencies. Survival time was calculated by Kaplan-Meier curves and the Log rank tests.

A $\mathrm{P}$ value $<0.05$ was considered statistically significant.

\section{Results}

\section{Patient Characteristics}

From January 2015 to December 2019, a total of 1667 patients diagnosed with MHBSs were hospitalized in our center. Among them, 296 subjects were excluded because they underwent radical surgeries, and 650 patients were excluded because they underwent palliative operations. In addition, 376 subjects were not included due to the lack of lab tests, and 139 patients lacked bile drainage records. Finally, a total of 206 patients were enrolled in this study, including 126 EBS patients and 80 PTBD patients, and 270 procedures were performed, including 173 EBS procedures and 97 PTBD procedures. The mean age of the subjects was $63 \pm 12$ years; the male:female ratio was 131:75; and primary cholangiocarcinoma accounted for $78.2 \%$ (161/206) of cases, gallbladder cancer for $8.7 \%$ (18/206), hepatocellular carcinoma for $6.8 \%$ (14/206), pancreatic cancer for $3.4 \%$ (7/206), and other metastases for $2.9 \%(6 / 206)$. Bismuth-Corlette classification grades I, II, III, and IV were noted in $65,60,48$, and 97 patients, respectively. The technical success rate was $99.6 \%$ (269/ 270), with one EBS attempt failing. Among patients in whom technical success was achieved, 64.9\% (172/265) achieved clinical success (4 patients without postprocedure bilirubin). The overall complication rate was $24.8 \%$ (67/ 270). The median cumulative survival duration was 183 days (range: 130 to 236 days).

\section{EBS versus PTBD in Patients with Bismuth Types III-IV}

The baseline characteristics were not different between the 2 groups (Table 1).

The bilirubin decline was higher in the PTBD group than in the EBS group (66.9 vs 36.7, p=0.006). The CS rates were $52.6 \%(50 / 95)$ and $67.4 \%(31 / 46)$ in the EBS and PTBD groups, respectively $(\mathrm{P}=0.097)$. The rate of early complications was $39.3 \%(57 / 145)$. The rates of overall early complications, cholangitis and hemorrhage were not different between the two groups (EBS vs PTBD $39.2 \%$ vs $39.6 \%$, $\mathrm{p}=0.962$, EBS vs PTBD $27.8 \%$ vs $29.2 \%, p=0.867$, EBS vs PTBD $3.1 \%$ vs $2.1 \%$, $\mathrm{p}=1.000)($ Table 2).

Table I The Baseline Characteristics Between EBS and PTBD in Patients with Bismuth Types III-IV

\begin{tabular}{|l|l|l|l|}
\hline Characteristic & $\begin{array}{l}\text { EBS } \\
(\mathbf{n = 9 7})\end{array}$ & $\begin{array}{l}\text { PTBD } \\
(\mathbf{n = 4 8 )}\end{array}$ & P value \\
\hline Age (years) & 62 & 58 & 0.099 \\
\hline Gender (n, \%) & $53(54.6 \%)$ & $29(60.4 \%)$ & \\
Male (n, \%) & $44(45.4 \%)$ & $19(39.6 \%)$ & \\
Female (n, \%) & & & 0.509 \\
\hline ECOG performance status & & & \\
(n, \%) & $5 I(52.6 \%)$ & $26(54.2 \%)$ & \\
2 & $46(47.4 \%)$ & $22(45.8 \%)$ & \\
3-4 & & & 0.867 \\
\hline TNM (n, \%) & $27(27.8 \%)$ & $14(29.2 \%)$ & \\
I-II & $70(72.2 \%)$ & $34(70.8 \%)$ & \\
III-IV & & & 0.677 \\
\hline Bismuth (n, \%) & $3 I(32.0 \%)$ & $17(35.4 \%)$ & \\
III & $66(68.0 \%)$ & $31(64.6 \%)$ & \\
IV & 181.75 & 227.80 & 0.078 \\
\hline TBIL ( $\mu \mathrm{mol} / \mathrm{L})$ & 81.90 & 83.65 & 0.979 \\
AST (U/L) & 68.30 & 88.15 & 0.329 \\
ALT (U/L) & 348.10 & 607.95 & 0.275 \\
CAI99 (U/mL) & 3.67 & 4.26 & 0.782 \\
CEA ( $\mu \mathrm{g} / \mathrm{L})$ & & & \\
\hline
\end{tabular}


Table 2 Efficacy and Safety Comparison Between EBS and PTBD in Patients with Bismuth Types III-IV

\begin{tabular}{|l|l|l|l|}
\hline & EBS (n=97) & PTBD (n=48) & p \\
\hline $\begin{array}{l}\text { *Clinical success }(\%, \\
\text { ratio) }\end{array}$ & $52.6 \%(50 / 95)$ & $67.4 \%(3 \mathrm{I} / 46)$ & 0.097 \\
TBIL decrease ( $\mu \mathrm{mol} / \mathrm{L})$ & 36.70 & & \\
Complication (\%, ratio) & $39.2 \%(38 / 97)$ & $39.6 \%(19 / 48)$ & 0.962 \\
Cholangitis (\%, ratio) & $27.8 \%(27 / 97)$ & $29.2 \%(14 / 48)$ & 0.867 \\
Hemorrhage (\%, ratio) & $3.1 \%(3 / 97)$ & $2.1 \%(\mathrm{I} / 48)$ & 1.000 \\
\hline
\end{tabular}

Notes: *Postprocedure TBIL was missing in 2 cases in each group.

Table 3 Comparison of the Baseline Characteristics Between Patients Who Underwent Bilateral and Unilateral Stenting

\begin{tabular}{|l|l|l|l|}
\hline Characteristic & $\begin{array}{l}\text { Unilateral } \\
(\mathbf{n = 8 0})\end{array}$ & $\begin{array}{l}\text { Bilateral } \\
(\mathbf{n = 4 7 )}\end{array}$ & P value \\
\hline Age (years) & 62 & 65 & 0.199 \\
\hline $\begin{array}{l}\text { Gender (n, \%) } \\
\text { Male }\end{array}$ & $48(60.0 \%)$ & $26(55.3 \%)$ & 0.606 \\
Female & $32(40.0 \%)$ & $21(44.7 \%)$ & \\
\hline ECOG performance & & & 0.629 \\
status (n, \%) & $39(48.8 \%)$ & $25(53.2 \%)$ & \\
3-4 & $41(51.2 \%)$ & $22(46.8 \%)$ & \\
\hline TNM (n, \%) & & & 0.636 \\
I-II & $24(30.0 \%)$ & $16(34.0 \%)$ & \\
III-IV & $56(70.0 \%)$ & $31(66.0 \%)$ & \\
\hline Bismuth (n, \%) & & & 0.178 \\
II & $24(30.0 \%)$ & $9(19.1 \%)$ & \\
III-IV & $56(70.0 \%)$ & $38(80.9 \%)$ & \\
\hline TBIL ( $\mu$ mol/L) & 157.90 & 196.10 & 0.057 \\
AST (U/L) & 67.50 & 120.50 & 0.003 \\
ALT (U/L) & 63.80 & 96.70 & 0.020 \\
CAI99 (U/mL) & 356.00 & 350.00 & 0.912 \\
CEA ( $\mu$ g/L) & 4.48 & 4.02 & 0.913 \\
\hline Stent types (n, \%) & $8(10.0 \%)$ & $4(8.5 \%)$ & 1.000 \\
Metal & $72(90.0 \%)$ & $43(91.5 \%)$ & \\
Plastic & & & \\
\hline
\end{tabular}

Table 5 The Clinical Success Rates in Unilateral and Bilateral Groups with Bismuth II-IV Scores

\begin{tabular}{|l|l|l|l|}
\hline \%(n) & Unilateral (n=79) & Bilateral (n=46) & $\mathbf{p}$ \\
\hline Bismuth II & $70.8 \%(I 7 / 24)$ & $77.8 \%(7 / 9)$ & 1.000 \\
Bismuth III & $59.1 \%(\mid 3 / 22)$ & $57.1 \%(4 / 7)$ & 1.000 \\
Bismuth IV & $30.3 \%(I 0 / 33)$ & $70.0 \%(2 I / 30)$ & 0.002 \\
\hline
\end{tabular}

\section{Unilateral versus Bilateral Stenting}

The baseline characteristics were matched between the two groups (Table 3).

A more significant bilirubin decrease was observed in the bilateral group than in the unilateral group (87.5 vs 18.9, $\mathrm{p}=0.001)$ (Table 4). Bilateral stenting yielded a higher rate of CS $(69.6 \%$ vs $50.6 \%, p=0.039)$, especially for patients with Bismuth type IV $(70.0 \%$ vs $30.3 \%$, $\mathrm{p}=0.002$ ) (Tables 4 and 5). The rates of overall complications (bilateral vs unilateral $40.4 \%$ vs $36.3 \%, \mathrm{p}=0.639$ ) and cholangitis (bilateral vs unilateral $31.9 \%$ vs $21.3 \%$, $\mathrm{p}=0.181$ ) were comparable between the two groups (Table 4).

\section{Survival}

No significant difference was found regarding survival time between EBS and PTBD (142 days vs 218 days, $\mathrm{p}=0.057$ ) (Figure 1) or between bilateral and unilateral stenting (172 days vs 106 days, $\mathrm{p}=0.371$ ) (Figure 2). A survival advantage was found with successful drainage (227 days vs 82 days, $\mathrm{p}<0.001$ ), lower TNM scores (I-II) (195 days vs 139 days, $\mathrm{p}=0.012$ ), and cholangiocarcinoma (184 days vs 84 days, $\mathrm{p}=0.001$ ) (Figures $3-5$ ).

\section{Discussion}

No consensus has been reached regarding the use of unilateral or bilateral drainage in patients with unresectable perihilar malignancies. In our study, bilateral stenting showed a higher clinical success rate than unilateral

Table 4 Efficacy and Safety Comparison Between Unilateral and Bilateral Stenting

\begin{tabular}{|l|l|l|l|}
\hline & Unilateral (n=80) & Bilateral (n=47) & p value \\
\hline *Clinical success (\%, ratio) & $50.6 \%(40 / 79)$ & $69.6 \%(32 / 46)$ & 0.039 \\
TBIL decrease $(\mu \mathrm{mol} / \mathrm{L})$ & 18.90 & 87.50 & $0.00 \mathrm{I}$ \\
Complication $(\%$, ratio) & $36.3 \%(29 / 80)$ & $40.4 \%(19 / 47)$ & 0.639 \\
Cholangitis (\%, ratio) & $21.3 \%(17 / 80)$ & $31.9 \%(I 5 / 47)$ & 0.181 \\
Pancreatitis (\%, ratio) & $12.5 \%(10 / 80)$ & $10.6 \%(5 / 47)$ & 0.754 \\
Hemorrhage (\%, ratio) & $5.0 \%(4 / 80)$ & $4.3 \%(2 / 47)$ & 0.849 \\
\hline
\end{tabular}

Note: *Postprocedure TBIL was missing in I case in each group. 


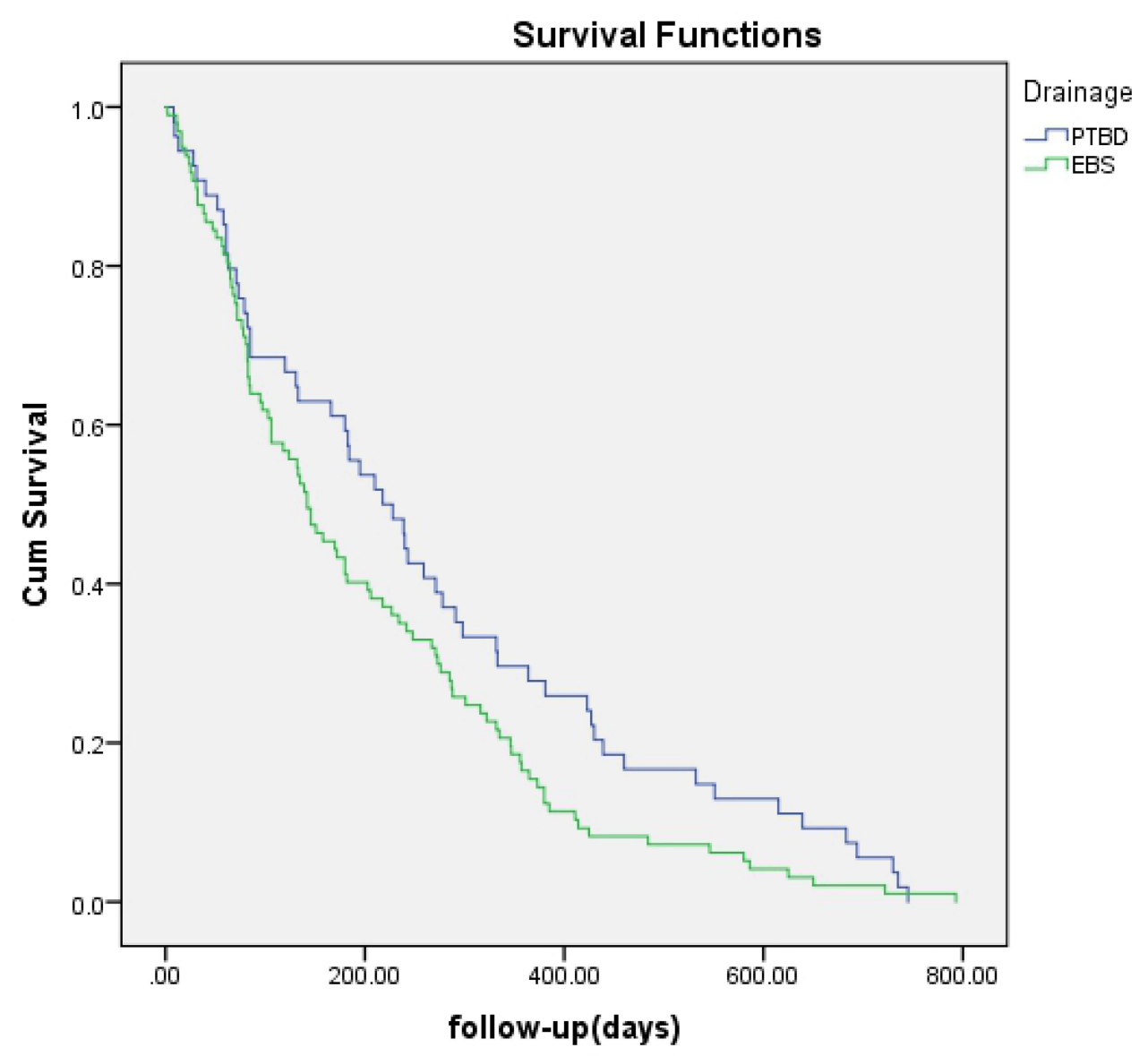

Figure I Survival time curve obtained by Kaplan-Meier analysis shows no difference between EBS and PTBD $(P=0.057)$.

stenting; this is in accordance with Lee et al's randomized controlled trial (RCT), which reported a clinical success rate of $95.3 \%$ in the bilateral group and $84.9 \%$ in the unilateral group $(\mathrm{p}=0.047) .^{5}$ According to Vienne et al's study, draining more than $50 \%$ of the liver volume is associated with more efficient drainage and longer patient survival. ${ }^{7}$ Hence, drainage of approximately $25-30 \%$ of liver volume by unilateral drainage may not be sufficient to satisfactorily reduce jaundice, and further bilateral stenting may be required. ${ }^{8}$ Notably, in our study, this advantage of bilateral drainage was more prominent with the Bismuth IV score. For high scores of Bismuth types, including Bismuth types III-IV, multiple stents are required to achieve adequate drainage. Given that it is technically difficult to place multiple stents through narrowed proximal bile ducts and failed attempts could increase the risk of cholangitis due to undrained opacified segments, ${ }^{9}$ we suggest that for patients with Bismuth grades II-IV, two or multiple instances of stenting should be performed by highly experienced endoscopists. Considering the limitations of a retrospective study, it is barely possible to distinguish between failed attempts of bilateral and unilateral drainage. Hence, technical success rates were not compared between the unilateral and bilateral groups. The overall rate of technical success in this study could be higher than it actually is and should be interpreted with caution.

It is well known that metal stents are superior to plastic stents for patients with unresectable MHBSs in terms of drainage adequacy and long-term results. ${ }^{10}$ However, plastic stents are preferred for preoperative drainage of MHBS, and metal stents may preclude curative surgery. ${ }^{11,12}$ In our study, plastic stents were inserted in most patients considering that surgeries had not been completely excluded before biliary drainage. According to Xia et al's study, bilateral drainage with metal stents outperformed that with plastic stents in terms of relieving jaundice. ${ }^{13}$ The utilization of plastic stents in our study may compensate for the effectiveness of bilateral drainage.

EBS and PTBD are well-established options for the palliation of patients with unresectable malignant obstructive jaundice. For palliation in MHBS of Bismuth types III 


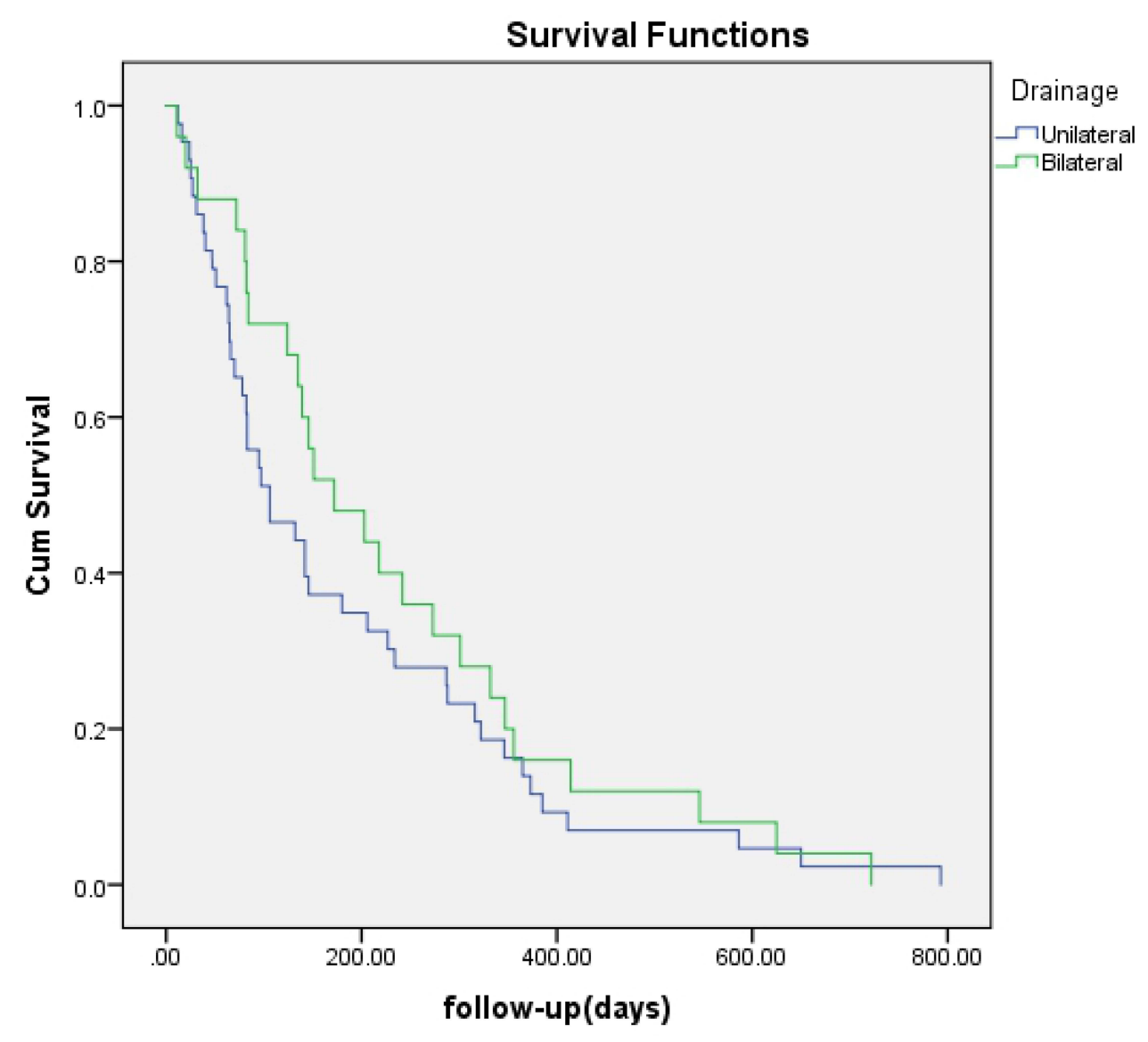

Figure 2 Survival time curve obtained by Kaplan-Meier analysis shows no difference between unilateral and bilateral drainage $(P=0.37 \mathrm{I})$.

and IV, it is unclear whether one approach is superior to the other. The European Society of Gastrointestinal Endoscopy (ESGE) suggests palliative drainage of malignant hilar strictures by means of PTBD or a combination of PTBD and EBS for Bismuth types III and IV to be modulated according to local expertise (weak recommendation, low-quality evidence). ${ }^{14}$ In the present study, for patients with Bismuth types III-IV, bilirubin declined more significantly in the PTBD group than in the EBS group, despite the absence of a significant difference in clinical success between the two groups. Higher rates of successful drainage by PTBD than EBS ( $\mathrm{OR}=2.53,95 \%$ $\mathrm{CI}=1.57$ to 4.08 ) were achieved in Moole's metaanalysis. ${ }^{15}$ The disadvantage of EBS may lead to the requirements of more procedures. A retrospective study compared the success rate and complications of EBS and PTBD in patients with hilar cholangiocarcinoma and reported that the initial EBS group received more drainage procedures than the PTBD group ( 2.8 vs $1.4, \mathrm{P}<0.01$ ), with 30 patients who received EBS (total of 90 patients) being converted to PTBD due to insufficient decompression. ${ }^{16}$
Furthermore, a subsequent study showed that inadequate drainage by EBS $(38 \%, 108 / 288)$ was associated with stenosis of Bismuth types III-IV. ${ }^{17}$ Compared with EBS, PTBD enables more precise lobar selection with guidance of ultrasound and CT scans, which would theoretically increase the success of biliary drainage. In our previous studies on obstructive jaundice rats, internal biliary drainage, which allows bile acid reappearance in the intestines, was more favorable than external drainage due to the pivotal role of bile acid in maintaining the integrity of the intestinal barrier, inhibiting bacterial growth, decreasing inflammation and improving immune function. ${ }^{18-20}$ However, in clinical studies, external drainage achieved by PTBD outperformed internal drainage by EBS. ${ }^{21}$ It is well known that unlike clinical studies, complications can be minimized in experimental studies. The advantages of internal drainage could be offset by its higher risk of complications in clinical scenarios.

However, compared with PTBD, EBS stenting can provide a drainage tube with a larger diameter and can avoid the inconvenience of the external lines and bags 


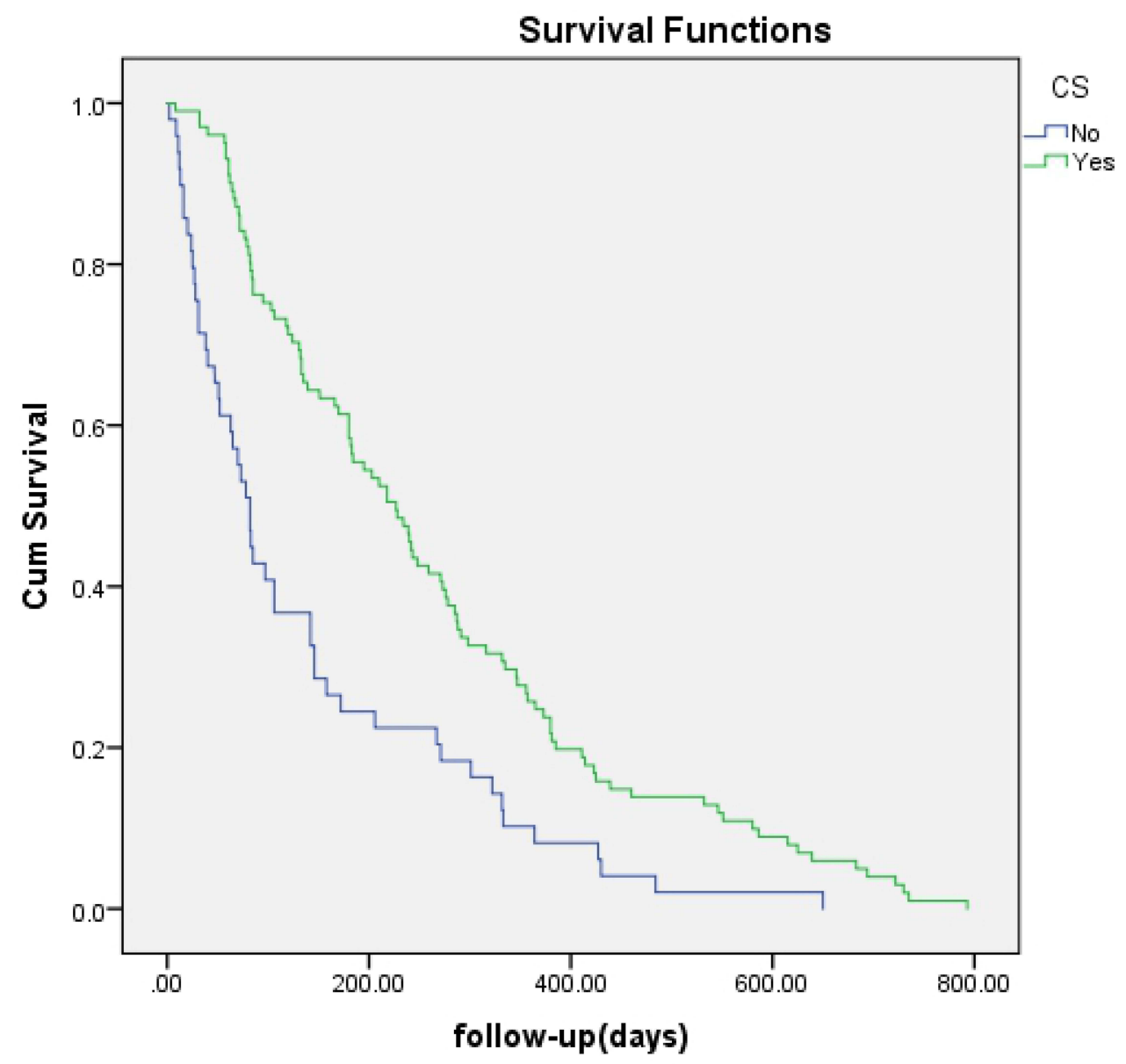

Figure 3 Kaplan-Meier analysis of survival between patients with or without clinical success. Cumulative survival rates were significantly higher in patients with CS (green line) $(P=0.000)$

associated with PTBD. Kongkam et al's study favors EBS over PTBD for MHBS due to the significantly lower rates of recurrent biliary obstruction with similar complication and mortality rates. ${ }^{22}$ Notably, in this study, stenting was performed by a combination of ERCP and endoscopic ultrasound techniques, whereas stenting was performed only by ERCP in our study. Moreover, EBS may outperform PTBD with respect to long-term results, and a recent RCT comparing the two modalities in patients with resectable hilar cholangiocarcinoma indicated higher mortality in the PTBD group. ${ }^{4}$ In our study, no survival benefit was observed in either group. Bilateral and unilateral stenting were also comparable in terms of survival time in our study. Lee et al's prospective RCT (including 47 bilateral procedures and 54 unilateral procedures) reported a similar survival duration in the two groups (bilateral group vs unilateral group: 270 days vs 178 days, $\mathrm{p}=0.053){ }^{5}$
In the present study, the overall survival duration was 183 days (range: 130 to 236 days), which is consistent with previous studies. ${ }^{2,3}$ Successful drainage was associated with a longer life span in our study. Clinical success can reduce bilirubin levels, preserve liver function, improve nutritional conditions and allow the administration of subsequent adjuvant therapies. These advantages may contribute to a better prognosis. Our data indicated that patients diagnosed with cholangiocarcinoma had a longer survival time than patients with other malignancies in the hilum, in agreement with Xia et al's study, which reported a better prognosis in patients with hilar cholangiocarcinoma (HR 0.419, $\mathrm{P}=0.009$ ). ${ }^{13}$ In our study, the considerably large proportion of gallbladder cancers may have contributed to the shorter life expectancies associated with the other types of tumors $(42.11 \%, 16 / 38)$. According to Pranculis et al's study (HR 31.029, 


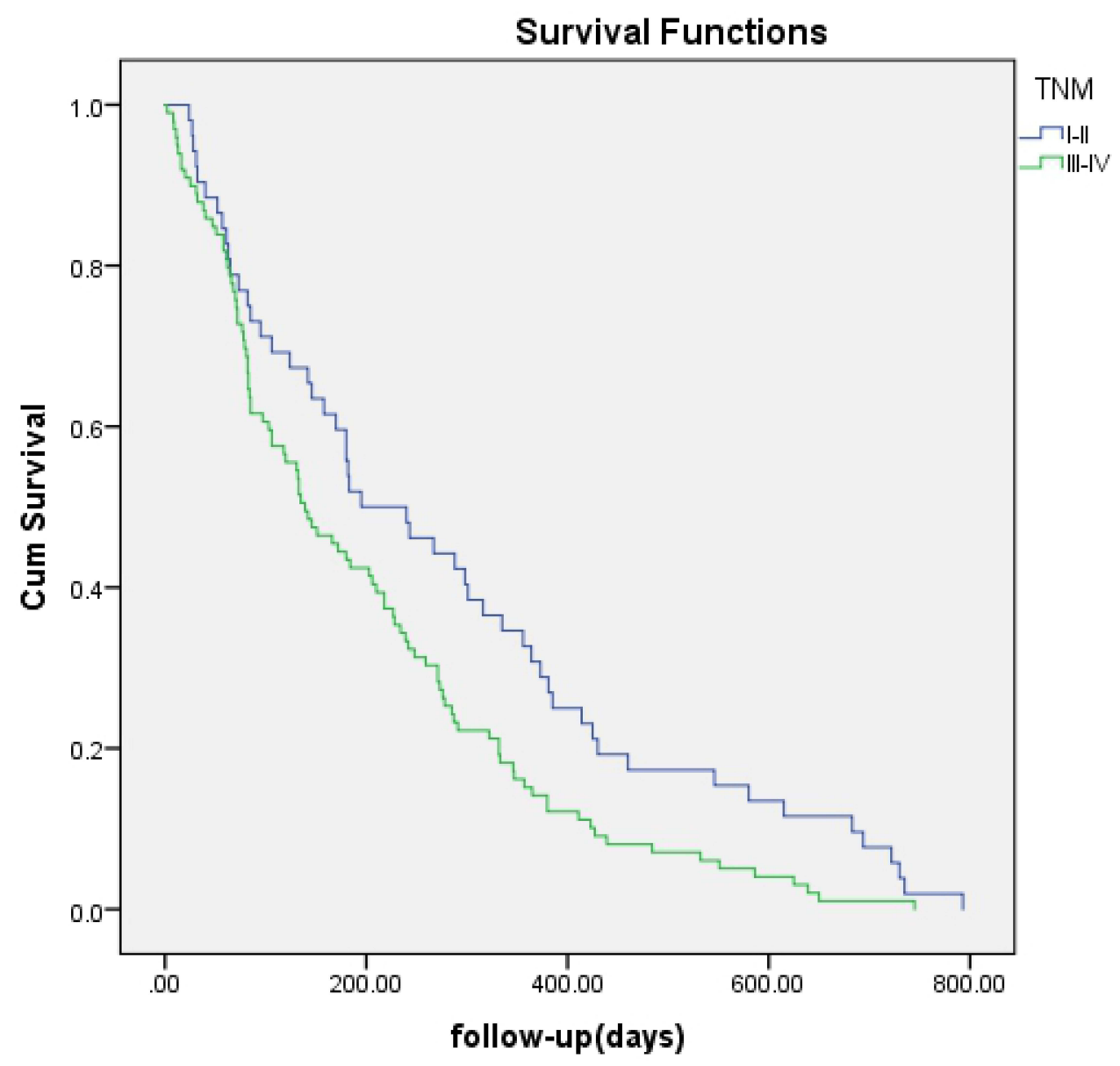

Figure 4 Kaplan-Meier analysis of survival between patients with TNM I-II and those with TNM III-IV. Cumulative survival rates were significantly higher in patients with TNM I-II (blue line) $(P=0.012)$.

$\mathrm{P}=0.012),{ }^{23}$ gallbladder cancer seemed to be a risk factor for poor prognosis. The poor prognosis of gallbladder cancer was also reported in several other studies. ${ }^{24-26}$

To date, optimal biliary drainage for patients with unresectable perihilar malignancies is still a controversial issue. Studies on palliative drainage for unresectable MHBS patients are sparse. A recent RCT was terminated prematurely because of the slow process of admission. ${ }^{27}$ We included a large number of patients with MHBSs to compare PTBD with EBD, as well as bilateral with unilateral stenting in aspect of short-term and long-term outcomes. Though, further properly powered RCTs addressing this topic are warranted to provide more efficient approaches for palliation.

There are several limitations of this study. First, because it is a retrospective study, it was inevitable to avoid bias and confounding factors. For instance, drainage protocols (EBS or PTBD, unilateral or bilateral stenting) were developed by endoscopists in our center based on their own experience and patients' conditions, which was difficult to track in the current study. Second, most patients received plastic stents, which is no longer a standard protocol. However, bilateral stenting showed superiority over unilateral stenting in successful drainage. Thus, the data should be interpreted with caution. Finally, EBS and PTBD were performed by highly experienced endoscopists at tertiary-care referral centers associated with a high prevalence of MHBS. Consequently, the technical success rates are expected to be higher and may not be able to be generalized to other hospitals. Further prospective multicenter studies evaluating optimal drainage strategies for high-grade MHBS are warranted.

\section{Conclusion}

In conclusion, for MHBS patients with high-grade Bismuth types, bilateral stenting is more likely than unilateral stenting to offer sufficient drainage with similar 


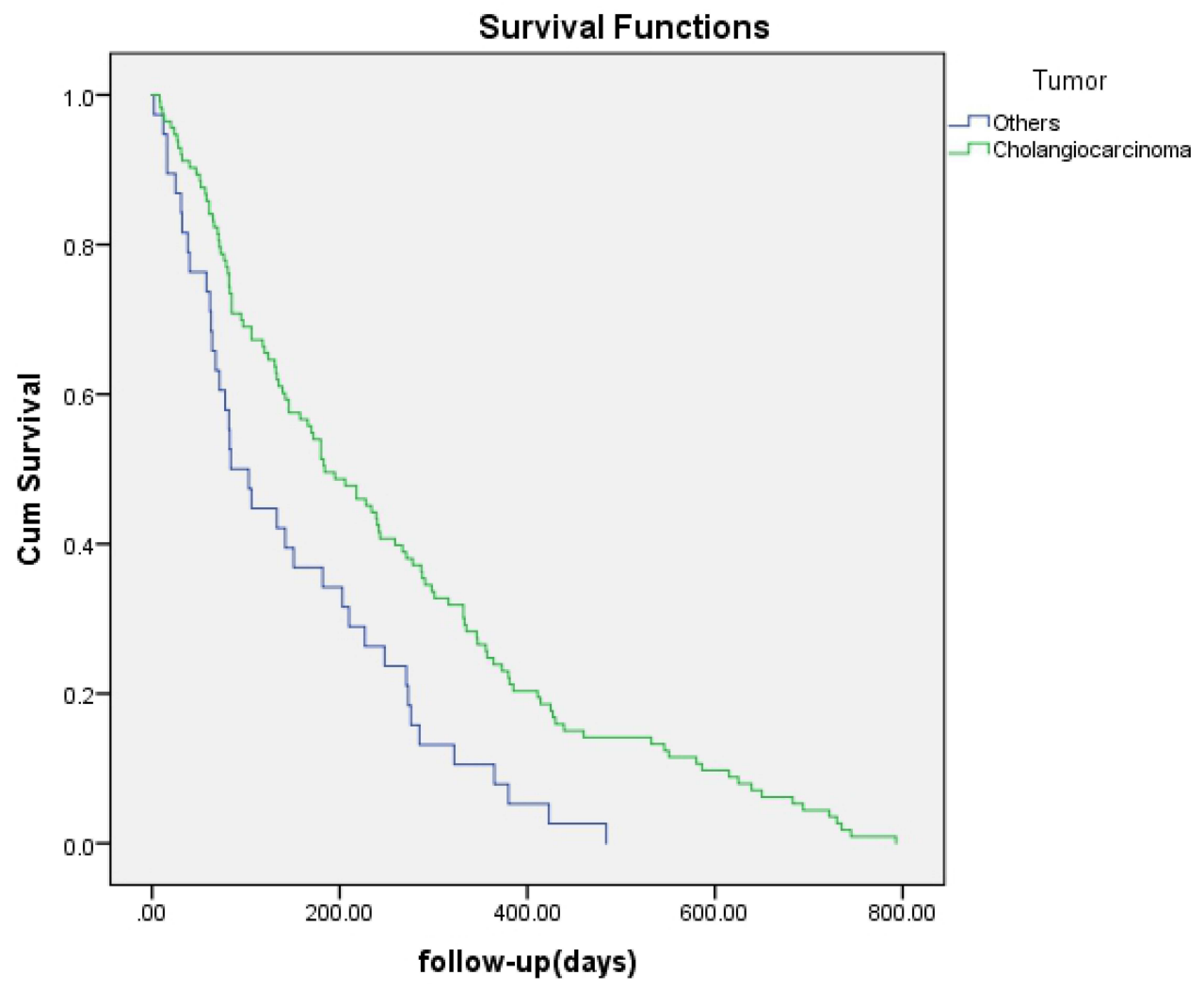

Figure 5 Kaplan-Meier analysis of survival between patients with cholangiocarcinoma and those with other tumors. Cumulative survival rates were significantly higher in patients with cholangiocarcinoma (green line) $(P=0.00 I)$.

rates of complications, and PTBD may be superior to EBS in mitigating cholestasis without increased adverse events. Further prospective studies should be conducted to assist practitioners with optimal drainage decisions for these patients.

\section{Abbreviations}

MHBSs, malignant hilar biliary strictures; EBS, endoscopic biliary stenting; PTBD, percutaneous transhepatic biliary drainage; TNM, tumor-node-metastasis; CT, computed tomography; MRI, magnetic resonance imaging; MRCP, magnetic resonance cholangiopancreatography; SBS, side-by-side; SIS, side-in-side; TS, technical success; CS, clinical success.

\section{Acknowledgments}

The funding source had no involvement in the design, analysis, or writing of this paper or in the decision to publish this work. Special thanks are expressed to the medical staff of the Gastroenterology Division, Medical School of Chinese PLA, Beijing, China.

\section{Disclosure}

The authors report no conflicts of interest related to this work.

\section{References}

1. Launois B, Reding R, Lebeau G, Buard JL. Surgery for hilar cholangiocarcinoma: french experience in a collective survey of 552 extrahepatic bile duct cancers. J Hepatobiliary Pancreat Surg. 2000;7 (2):128-134. doi:10.1007/s005340050166

2. Hemming AW, Reed AI, Fujita S, Foley DP, Howard RJ. Surgical management of hilar cholangiocarcinoma. Ann Surg. 2005;241 (5):693-702. doi:10.1097/01.sla.0000160701.38945.82

3. Nishio H, Nagino M, Nimura Y. Surgical management of hilar cholangiocarcinoma: the Nagoya experience. HPB (Oxford). 2005;7 (4):259-262. doi:10.1080/13651820500373010

4. Coelen RJS, Roos E, Wiggers JK, et al.Endoscopic versus percutaneous biliary drainage in patients with resectable perihilar cholangiocarcinoma: a multicentre, randomised controlled trial. $J$ Lancet Gastroenterol Hepatol. 2018;3(10):681-690.

5. Lee TH, Kim TH, Moon JH, et al. Bilateral versus unilateral placement of metal stents for inoperable high-grade malignant hilar biliary strictures: a multicenter, prospective, randomized study (with video). Gastrointest Endosc. 2017;86(5):817-827.

6. Chaiteerakij R, Harmsen WS, Marrero CR, et al. A new clinically based staging system for perihilar cholangiocarcinoma. Am J Gastroenterol. 2014;109(12):1881-1890. doi:10.1038/ ajg. 2014.327 
7. Vienne A, Hobeika E, Gouya H, et al. Prediction of drainage effectiveness during endoscopic stenting of malignant hilar strictures: the role of liver volume assessment. Gastrointest Endosc. 2010;72 (4):728-735. doi:10.1016/j.gie.2010.06.040

8. Mukai T, Yasuda I, Nakashima M, et al. Metallic stents are more effificacious than plastic stents in unresectable malignant hilar biliary strictures: a randomized controlled trial. J Hepatobiliary Pancreat Sci. 2013;20(2):214-222. doi:10.1007/s00534-012-0508-8

9. Deviere J, Baize M, de Toeuf J, Cremer M. Long-term followup of patients with hilar malignant stricture treated by endoscopic internal biliary drainage. Gastrointest Endosc. 1988;34(2):95-101. doi:10.1016/S0016-5107(88)71271-7

10. Sangchan A, Kongkasame W, Pugkhem A, et al. Efficacy of metal and plastic stents in unresectable complex hilar cholangiocarcinoma: a randomized controlled trial. Gastrointest Endosc. 2012;76 (1):93-99. doi:10.1016/j.gie.2012.02.048

11. Kawashima H, Itoh A, Ohno E, et al. Preoperative endoscopic nasobiliary drainage in 164 consecutive patients with suspected perihilar cholangiocarcinoma: a retrospective study of efficacy and risk factors related to complications. Ann Surg. 2013;257(1):121-127. doi:10.1097/SLA.0b013e318262b2e9

12. Grünhagen DJ, Dunne DFJ, Sturgess RP, et al. Metal stents: a bridge to surgery in hilar cholangiocarcinoma. HPB (Oxford). 2013;15 (5):372-378. doi:10.1111/j.1477-2574.2012.00588.X

13. Xia MX, Pan YL, Cai XB, et al. Comparison of endoscopic bilateral metal stent drainage with plastic stents in the palliation of unresectable hilar biliary malignant strictures: large multicenter study. Dig Endosc. 2021;33(1):179-189. doi:10.1111/den.13680

14. Tringali A, Papanikolaou IS, Blero D,et al.Endoscopic biliary stenting: indications, choice of stents, and results: European Society of Gastrointestinal Endoscopy (ESGE) Clinical Guideline - Updated October 2017. J Endoscopy. 2018;50(9):910-930

15. Moole H, Dharmapuri S, Duvvuri A, et al. Endoscopic versus percutaneous biliary drainage in palliation of advanced malignant hilar obstruction: a meta-analysis and systematic Review. Can $J$ Gastroenterol Hepatol. 2016;2016:1-8. doi:10.1155/2016/4726078

16. Kloek JJ, van der Gaag NA, Aziz Y, et al. Endoscopic and percutaneous preoperative biliary drainage in patients with suspected hilar cholangiocarcinoma. J Gastrointest Surg. 2010;14(1):119-125. doi:10.1007/s11605-009-1009-1

17. Wiggers JK, Groot Koerkamp B, Coelen RJ, et al. Preoperative biliary drainage in Perihilar Cholangiocarcinoma: identifying patients who require percutaneous drainage after failed endoscopic drainage. Endoscopy. 2015;47(12):1124-1131. doi:10.1055/s-0034-1392559
18. Wang ZK, Xiao JG, Huang XF, Gong YC, Li W. Effect of biliary drainage on inducible nitric oxide synthase, CD14 and TGR5 expression in obstructive jaundice rats. World J Gastroenterol. 2013;19 (15):2319-2330. doi:10.3748/wjg.v19.i15.2319

19. Wu L, Li W, Wang Z, Yuan Z, Hyder Q. Bile acid-induced expression of farnesoid $\mathrm{X}$ receptor as the basis for superiority of internal biliary drainage in experimental biliary obstruction. Scand J Gastroenterol. 2013;48(4):496-503. doi:10.3109/00365521.2012.763173

20. Meng Y, Gong YC, Dou Y, Li W. Changes of serum cytokines and expression of inducible nitric oxide synthase mRNA by Kupffer cells after relief from obstructive jaundice in rats. J Gastroenterol Hepatol. 2009;24(6):1064-1069. doi:10.1111/j.1440-1746.2008.05746.x

21. Tian XP, Zhang ZX, Li W. Internal drainage versus external drainage in palliation of malignant biliary obstruction: a meta-analysis and systematic review. Arch Med Sci. 2020;16(4):752-763. doi:10.5114/ aoms.2020.94160

22. Kongkam P, Orprayoon T, Boonmee C, et al.ERCP plus endoscopic ultrasound-guided biliary drainage versus percutaneous transhepatic biliary drainage for malignant hilar biliary obstruction: a multicenter observational open-label study. Endoscopy. 2021;53(1):55-62. doi:10.1055/a-1195-8197

23. Pranculis A, Kievišas M, Kievišienè L, et al. Percutaneous transhepatic biliary stenting with uncovered self-expandable metallic stents in patients with malignant biliary obstruction - efficacy and survival analysis. Pol J Radiol. 2017;82(82):431-440. doi:10.12659/ PJR.901785

24. Vij JC, Govil A, Chaudhary A, Gulati R, Mehta S, Ganguli S. Endoscopic biliary endoprosthesis for palliation of gallbladder carcinoma. Gastrointest Endosc. 1996;43(2):121-123. doi:10.1016/ S0016-5107(06)80112-4

25. Gao DJ, Hu B, Ye X, et al. Metal versus plastic stents for unresectable gallbladder cancer with hilar duct obstruction. Dig Endosc. 2017;29(1):97-103. doi:10.1111/den.12700

26. De Palma GD, Galloro G, Siciliano S, et al. Unilateral versus bilateral endoscopic hepatic duct drainage in patients with malignant hilar biliary obstruction: results of a prospective, randomized, and controlled study. Gastrointest Endosc. 2001;53(6):547-553. doi:10.1067/ mge.2001.113381

27. Elmunzer BJ, Smith ZL, Tarnasky P, et al. An unsuccessful randomized trial of percutaneous vs endoscopic drainage of suspected malignant hilar obstruction. Clin Gastroenterol Hepatol. 2020;23.

\section{Publish your work in this journal}

Cancer Management and Research is an international, peer-reviewed open access journal focusing on cancer research and the optimal use of preventative and integrated treatment interventions to achieve improved outcomes, enhanced survival and quality of life for the cancer patient.
The manuscript management system is completely online and includes a very quick and fair peer-review system, which is all easy to use. Visit http://www.dovepress.com/testimonials.php to read real quotes from published authors. 